\title{
Comparison of neuroregeneration in central nervous system and peripheral nervous system
}

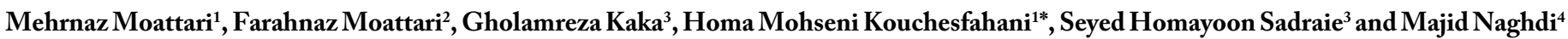 \\ ${ }^{1}$ Department of Animal Biology, Faculty of Biological Science, Kharazmi University, Iran \\ ${ }^{2}$ Department of Animal Biology, Faculty of Biological Science, Kharazmi University, Iran \\ ${ }^{3}$ Neuroscience Research Center, Baqiyatallah University of Medical Sciences, Iran \\ ${ }^{4}$ Fasa University of Medical Science, Iran
}

\begin{abstract}
The nervous system is divided into two parts: the central nervous system, which consists of the brain and spinal cord, and the peripheral nervous system, which consists of cranial and spinal nerves along with their associated ganglia. Neurodegeneration or nerve repair refers to the regrowth or repair of nervous tissues, cells or cell products. Such mechanisms may include generation of new neurons, glia, axons, myelin, or synapses. Neurodegeneration differs between the peripheral nervous system (PNS) and the central nervous system (CNS) by the functional mechanisms and especially the extent and speed. While the peripheral nervous system has an intrinsic ability for repair and regeneration, the central nervous system is, for the most part, incapable of self-repair and regeneration. Here, we have an overview on the differences between peripheral nervous system and central nervous system.
\end{abstract}

\section{Introduction}

Nervous system injuries affect over 90,000 people every year [1] It is estimated that spinal cord injuries alone affect 10,000 each year [2]. Peripheral nerve injury is prevalent clinically. The rate of peripheral nerve is assessed between 13 and 23 per100,000 persons per year injuries in developed countries [3].

When a peripheral nerve injured it undergoes an initial phase of "Wallerian degeneration" as follows. Injury to the peripheral nervous system immediately elicits the migration of phagocytes, Schwann cells (produce myelin in PNS), and macrophages to the lesion site to clear away debris such as damaged tissue. the serum-derived opsonin stimulates the macrophages to remove the myelin residues. The rapid removal of myelin speeds up axonal repair [4]. When a nerve axon is severed, the end still attached to the cell body is labeled the proximal segment, while the other end is called the distal segment. After injury, the proximal end swells and experiences some retrograde degeneration, but once the debris is cleared, it begins to sprout axons and the presence of growth cones can be detected. The proximal axons are able to regrow as long as the cell body is intact, and they have made contact with the Schwann cells in the endoneurial channel or tube. The distal segment, however, experiences Wallerian degeneration within hours of the injury; the axons and myelin degenerate, but the endoneurium remains. In the later stages of regeneration, the remaining endoneurial tube directs axon growth back to the correct targets [5]. To repair, axons must have the inherent ability to grow, the distal environment must support the growth again and target tissues must accept new axons. During a long time without axon repair, the distal end of the nerve and target tissues are not chronically digested, and the neurons suffer from axonal exclusion, that is, their axon does not communicate with the target tissue. This condition causes apoptosis of the neurons and chronic non-nerve and chronic osteotomy prevents repair. In a healthy nerve, Schwann cells keep the blade intact osteotomy prevents repair [4]. In a healthy nerve, during Wallerian degeneration, Schwann cells grow in ordered columns along the endoneurial tube, creating a band of Büngner that protects and preserves the endoneurial channel. Also, macrophages and Schwann cells release neurotrophic factors that enhance re-growth [5]. Also, distal damage is better than proximal damage due to the need for shorter distances to reach their target tissue [4].

Unlike peripheral nervous system injury, injury to the central nervous system is not followed by extensive regeneration. It is limited by the inhibitory influences of the glial and extracellular environment. Slower degeneration of the distal segment than that which occurs in the peripheral nervous system also contributes to the inhibitory environment because inhibitory myelin and axonal debris are not cleared away as quickly. All these factors contribute to the formation of what is known as a glial scar, which axons cannot grow across. The proximal segment attempts to regenerate after injury, but its growth is hindered by the environment. It is important to note that central nervous system axons have been proven to regrow in permissive environments; therefore, the primary problem to central nervous system axonal regeneration is crossing or eliminating the inhibitory lesion site [6]. Glial cell scar formation is induced following damage to the nervous system. In the central nervous system, this glial scar formation significantly inhibits nerve regeneration, which leads to a loss of function. Several families of molecules are released that promote and drive glial scar formation. For instance, transforming growth

*Correspondence to: Homa Mohseni Kouchesfahani, Department of Animal Biology, Faculty of Biological Science, Kharazmi University, Tehran, Iran, Tel: 982126127286, E-mail: homamohseni123@gmail.com

Key words: peripheral nervous system, central nervous system, spinal cord, axons Received: July 30, 2018; Accepted: August 15, 2018; Published: August 20, 2018 
factors $\beta-1$ and -2 , interleukins, and cytokines which play a role in the initiation of scar formation. The accumulation of reactive astrocytes at the site of injury and the up regulation of molecules that are inhibitory for neurite outgrowth contribute to the failure of neurodegeneration [7].

A comparison of the two systems confirms that the central nervous system intruder is the biggest challenge for the restoration of its axons and has led to the discovery of multiple factors in the growth factor in the peripheral nervous system or inhibition of growth in the central nervous system. This inhibition is because of the hostile, nonpermissive growth environment is, in part, created by the migration of myelin-associated inhibitors, astrocytes (support neurons in CNS), oligodendrocytes (produce myelin in CNS), oligodendrocyte precursors, and microglia (participate in phagocytosis). Myelin produced by oligodendrocyte and Schwann cells have both inhibitory molecules, but myelin residues in the peripheral nervous system are further eradicated after damage in comparison to the central nervous system. In the central nervous system, oligodendrocytes do not remove myelin residues. Failure to break the blood-brain barrier prevents serum opsonin and peripheral macrophages from appearing to distal white matter pathways, and the presence of myelin residues in this system prevents axonal regeneration. The environment within the CNS, especially following trauma, counteracts the repair of myelin and neurons. Growth factors are not expressed or re-expressed; for instance, the extracellular matrix is lacking laminins. Glial scars rapidly form, and the glia actually produce factors that inhibit remyelination and axon repair. For instance, chondroitin sulfate proteoglycan (CSPGs), astrocytes up regulate the production of chondroitin sulfate proteoglycans.

\section{Keratan sulfate proteoglycans or keratosulfate (KSPG)}

Reactive astrocytes up regulate the chondroitin sulfate proteoglycans as part of glial scar formation and inhibit neurite outgrowth extension, limiting nerve regeneration. Proteins of oligodendritic or glial debris origin that influence neurodegeneration including NOGO: inhibitor of remyelination in the CNS; antagonizing this inhibitor results in improved remyelination, as it is involved in the RhoA pathway. NI35 a non-permissive growth factor from myelin [6], MAG (Myelinassociated glycoprotein): acts via the receptors NgR2, GT1b, NgR1, p75, TROY and LINGO1, OMgp (Oligodendrocyte Myelin glycoprotein), Ephrin B3: inhibits remyelination, Sema 4D (Semaphorin 4D): inhibits remyelination [8].

Sema 3A (Semaphorin 3A) is present in the scar that forms in both central nervous system [9] and peripheral nerve injuries and contributes to the outgrowth-inhibitory properties of these scars [10]. The axons themselves also lose the potential for growth with age, due to a decrease in GAP 43(growth associated protein 43) expression among others [11].

Another problem is that the morphology and functional properties of central nervous system neurons are highly complex, for this reason a neuron cannot be functionally replaced by one of another type (Llinas law) [7].

Neurodegeneration in the peripheral nervous system (PNS) occurs to a significant degree [6]. Axonal sprouts form at the proximal stump and grow until they enter the distal stump. The growth of the sprouts is governed by chemotactic factors secreted from Schwann cells (neurolemmocytes). In the central nervous system, the outward growth of axons stops at the site of damage that consists of reactive astrocytes and microglia. In contrast, no glial oscillation is formed in the peripheral nervous system, and Bunger bands formed by Schawann cells help to repair. In the peripheral nervous system, the rate of degeneration makes the extracellular medium possible to repair axon, while in the central nervous system, slow degeneration prevents axonal repair due to the presence of myelin inhibitors [4]. Nerve repair is more effective in the peripheral nervous system. Although peripheral axons can repair and form functional relationships, several factors such as the patient's age, pre-intervention delay and type of damage determine the outcome of postoperative performance. Given the advances in treatment in environmental damage models, clinical failure is a surprise. In fact, several factors contribute to the conversion of findings from rodent-toclinic studies. First, axonal repair occurs longer than rodents. As axon growth depends on the production and transportation of the material from the cell, the repair speed is like the slow transfer of axon, which is approximately 1 to $3 \mathrm{~mm}$ per day. The rate of recovery in humans is inherently slower than rodents. Human axon growth rates can reach $1 \mathrm{~mm} /$ day in small nerves and $5 \mathrm{~mm} /$ day in large nerves [6].

Several ways can help repair and contribute to clinical success, such as increasing neuron survival, improving the outbreak of axonal axon, and the inherent ability of axons to cope with inhibitory messages; preventing Schwann cell and atrophy or substitution of degenerated tissues with appropriate scaffolds and prevention of atrophy of the target tissue [12].

\section{Conclusion}

As a result of this high incidence of neurological injuries, nerve regeneration and repair, a subfield of neural tissue engineering, is becoming a rapidly growing field dedicated to the discovery of new ways to recover nerve functionality after injury. There is currently no treatment for recovery of human nerve function after injury to the central nervous system [12]. In addition, multiple attempts at nerve re-growth across the PNS-CNS transition have not been successful [12]. There is simply not enough knowledge about regeneration in the central nervous system. In addition, although the peripheral nervous system has the capability for regeneration, much research still needs to be done to optimize the environment for maximum regrowth potential. Researchers have argued that neurons in the central nervous system have less inherent ability to repair, but the theory was challenged by the discovery that central nervous system nerves can grow in the graft of the peripheral nerves. Understanding the significant differences in the repair of these two systems help improve recovery of central nervous system and chronic non-inflammation in the peripheral nervous system. Finally, neurodegeneration is important clinically, as it is a part of the pathogenesis of many diseases, including multiple sclerosis.

\section{References}

1. Stabenfeldt SE, García AJ, LaPlaca MC (2006) Thermoreversible lamininfunctionalized hydrogel for neural tissue engineering. J Biomed Mater Res Part A 77: 718-725.

2. Prang P, Müller R, Eljaouhari A, Heckmann K, Kunz W, et al. (2006) The promotion of oriented axonal regrowth in the injured spinal cord by alginate-based anisotropic capillary hydrogels. Biomaterials 27: 3560-3569.

3. Moattari M, Kouchesfehani HM, Kaka G, Sadraie SH, Naghdi M, et al. (2018) Chitosan-film associated with mesenchymal stem cells enhanced regeneration of peripheral nerves: A rat sciatic nerve model. $J$ Chem Neuroanat 88: 46-54.

4. Vargas ME, Barres BA (2007) Why is Wallerian degeneration in the CNS so slow? Annu Rev Neurosci 30: 153-179. [Crossref]

5. Shen Y, Nie H (2018) Wallerian degeneration of the bilateral middle cerebellar peduncles secondary to pontine infarction. Neurol Sci 39: 961-963.

6. Yiu G, He Z (2006) Glial inhibition of CNS axon regeneration. Nat Rev Neurosci 7: 617-627. [Crossref] 
7. Zhang H, Uchimura K, Kadomatsu K (2006) Brain keratan sulfate and glial scar formation. Ann N Y Acad Sci 1086: 81-90. [Crossref]

8. Bradbury EJ, McMahon SB (2006) Spinal cord repair strategies: why do they work? Nat Rev Neurosci 7: 644-653. [Crossref]

9. Mecollari V, Nieuwenhuis B, Verhaagen J (2014) A perspective on the role of class III semaphorin signaling in central nervous system trauma. Front Cell Neurosci 8: 328. [Crossref]
10. Tannemaat MR, Korecka J, Ehlert EME, Mason MRJ, van Duinen SG, et al. (2007) Human neuroma contains increased levels of semaphorin 3A, which surrounds nerve fibers and reduces neurite extension in vitro. J Neurosci 27: 14260-14264.

11. Ueha R, Shichino S, Ueha S, Kondo K, Kikuta S, et al. (2018) Reduction of proliferating olfactory cells and low expression of extracellular matrix genes are hallmarks of the aged olfactory mucosa. Front Aging Neurosci 10: 86.

12. Recknor JB (2006) Nerve regeneration: tissue engineering strategies. Tissue Eng Artif organs: 48

Copyright: $\mathbb{C} 2018$ Moattari M. This is an open-access article distributed under the terms of the Creative Commons Attribution License, which permits unrestricted use, distribution, and reproduction in any medium, provided the original author and source are credited. 